\title{
Different Impacts of Short-Chain Fatty Acids on Saturated and Polyunsaturated Fatty Acid Biosynthesis in Aurantiochytrium sp. SD116
}

\author{
Xiaojin Song, ${ }^{*}, \dagger, \|$ Yanzhen Tan, ${ }^{\dagger}, \|$ Yajun Liu, ${ }^{\dagger}$ Jingtao Zhang, $^{\dagger}$ Guanglei Liu, ${ }^{\dagger}$ Yingang Feng, ${ }^{\dagger}$ \\ and Qiu Cui*,†,
} ${ }^{\dagger}$ Shandong Provincial Key Laboratory of Energy Genetics and ${ }^{\ddagger}$ Key Laboratory of Biofuels, Qingdao Institute of Bioenergy and
Bioprocess Technology, Chinese Academy of Sciences, Qingdao 266101, Shandong, People’s Republic of China

\section{Supporting Information}

ABSTRACT: Aurantiochytrium is an important docosahexaenoic acid (DHA) producer containing two kinds of fatty acid synthesis pathways, that is, the fatty acid synthase pathway (FAS) for saturated fatty acid synthesis and the polyketide synthase pathway (PKS) for polyunsaturated fatty acid synthesis. To understand the regulation mechanism between the two pathways, the impacts of six short-chain fatty acids on the fatty acid synthesis of Aurantiochytrium sp. SD116 were studied. All short-chain fatty acids showed little effect on the cell growth, but some of them significantly affected lipid accumulation and fatty acid composition. Pentanoic acid and isovaleric acid greatly inhibited the synthesis of saturated fatty acids, whereas the polyunsaturated fatty acid synthesis was not affected. Analysis of malic enzyme activity, which supplied NADPH for saturated fatty acids biosynthesis, indicated that the two fatty acid synthesis pathways can utilize different substrates and possess independent sources of NADPH.

KEYWORDS: Aurantiochytrium, docosahexaenoic acid, fatty acid synthase, malic enzyme, polyketide synthase

\section{INTRODUCTION}

Docosahexaenoic acid (DHA; C22:6, n-3; (4Z,7Z,10Z,13Z,16Z,19Z)-docosa-4,7,10,13,16,19-hexaenoic acid) has received worldwide attention due to its beneficial effects for humans, such as improving neural and retinal development in infants and reducing risk factors involved in hypertension, cardiovascular diseases, arthritis, and arteriosclerosis. ${ }^{1-3}$ Oceanic fish and fish oil products are the typical dietary sources of $\mathrm{DHA}^{4}$ However, because of emerging concerns over the sustainability of marine resources and the levels of environmental contaminants present in fish, major efforts have been made to identify or create alternative sources of DHA. At present, the major alternative sources for commercial DHA production are Aurantiochytrium sp. and Crypthecodinium cohnii. ${ }^{5}$ For example, the product Life's DHA by DSM Co. is a commercial DHA from algae Aurantiochytrium.

Aurantiochytrium sp. is heterotrophic marine thraustochytrids with higher growth rate and more DHA production than $C$. cohnii. $^{6-8}$ During the past 20 years, studies dealing with this organism mainly focused on the effects of cultivation conditions, cultivation styles, and medium compositions including carbon, nitrogen sources, inorganic salt, and mineral addition on DHA productivity. ${ }^{9-13}$ However, the mechanism of fatty acid synthesis and lipid accumulation is still not well investigated.

Two kinds of fatty acid synthesis pathways, that is, the fatty acid synthase (FAS) pathway and the polyketide synthase (PKS) pathway, were discovered in Aurantiochytrium sp. ${ }^{14}$ DHA and omega-6 docosapentaenoic acid (DPA; C22:5, n-6; $(7 Z, 10 Z, 13 Z, 16 Z, 19 Z)$-docosa-7,10,13,16,19-pentaenoic acid $)^{15}$ are mainly synthesized via the PKS pathway, ${ }^{16}$ and other fatty acids are synthesized via the FAS pathway. ${ }^{17}$ Sufficient supply of acyl-CoA, ATP, and NADPH, employed as substrate, energy source, and reductant, respectively, are three key factors to engender lipid accumulation. ${ }^{18}$

According to former studies, linear or branched carboxylic acids, including short-chain fatty acids (SCFAs), could supply the acyl-CoA as the starter units for both the FAS and PKS pathways. ${ }^{19}$ The production of NADPH for fatty acid biosynthesis is dependent on malic enzyme (ME). ${ }^{20,21}$ However, previous conclusions were all based on microorganisms possessing a single pathway system, the FAS or PKS pathway system, and the reported studies on the PKS pathway were mainly for the antibiotic biosynthesis rather than the polyunsaturated fatty acid (PUFA) biosynthesis. Thus, it is not clear how the two pathways are regulated when both FAS and PKS pathways coexist for fatty acid biosynthesis in one microorganism, for example, Aurantiochytrium. Research on the regulation of the two pathways in Aurantiochytrium could provide clues to improve DHA production utilizing the organism in the food industry.

Previous research has shown SCFAs could be utilized by some marine alga, and the incorporation of SCFA can provide some insight into the fatty acid biosynthesis mechanism. ${ }^{22}$ In this study, the impact of different SCFAs including acetic acid, propanoic acid, isobutyric acid (2-methylpropanoic acid),

Received: July 18, 2013

Revised: September 21, 2013

Accepted: September 22, 2013

Published: September 23, 2013 
Table 1. Impact of Short-Chain Fatty Acids on the Biomass, Glucose Consumption, Acid Consumption, and Percent Conversion of Carbon after the $84 \mathrm{~h}$ Fermentation with Total Volume of $3.5 \mathrm{~L}$

$\begin{array}{lcccc}\text { experiment } & \text { biomass }(\mathrm{g}) & \text { glucose consumption }(\mathrm{g}) & \text { acid consumption }(\mathrm{mL}) & \text { \% conversion of carbon }(\mathrm{g} \mathrm{DCW} / \mathrm{mol} \text { carbon })^{a} \\ \text { control } & 368.8 & 1062.8 & 33 & 10.4 \\ \text { acetic acid } & 377.3 & 650.7 & 327 & 7.6 \\ \text { propanoic acid } & 365.3 & 626.4 & 275 & 8.3 \\ \text { butanoic acid } & 389.2 & 691.8 & 282 & 7.8 \\ \text { isobutyric acid } & 347.7 & 619.7 & 241 & 7.8 \\ \text { pentanoic acid } & 331.1 & 607.3 & 249 & 7.9 \\ \text { isovaleric acid } & 348.8 & 643.1 & 2.9\end{array}$

${ }^{a_{\text {Total }}}$ mole number of carbon is the sum of carbon mole numbers in glucose consumption and carbon mole numbers in acid consumption.

butanoic acid, isovaleric acid (3-methylbutanoic acid), and pentanoic acid on the fatty acid biosynthesis in Aurantiochytrium was studied. Six SCFAs were individually fed into medium in an independent treatment group during the fermentation to supply various acyl-CoAs, and the NADPH formation of Aurantiochytrium sp. SD116 was analyzed by detecting the changes of the key ME activities at different stages in each treatment. The purpose of this study is to try to understand how the substrates (acyl-CoA and NADPH) are distributed and regulated between the FAS and PKS pathways in Aurantiochytrium sp. SD116. The change of the fatty acid composition and ME activities demonstrated the different substrate utilizations and NADPH sources of the FAS and PKS pathways during the fatty acid biosynthesis in Aurantiochytrium sp. SD116. These findings present new insight into the metabolic regulation of saturated fatty acid (SFA) and DHA biosynthesis in this organism, which could provide new clues to improve the DHA production by Aurantiochytrium sp.

\section{MATERIALS AND METHODS}

Microorganism. Aurantiochytrium sp. SD116 (CGMCC no. 6208), isolated from seawater and stored in the China General Microbiological Culture Collection Center (CGMCC), ${ }^{7}$ was used in the present study. This strain was preserved in $20 \%$ (v/v) glycerol at $-80{ }^{\circ} \mathrm{C}$.

Culture Conditions. The seed culture medium contained $60 \mathrm{~g} \mathrm{~L}^{-1}$ glucose and $20 \mathrm{~g} \mathrm{~L}^{-1}$ yeast extract, which were dissolved in artificial seawater. This medium also contained trace elements in a prepared solution $\left(1 \mathrm{~mL} \mathrm{~L}^{-1}\right)$ and vitamin solution $\left(2 \mathrm{~mL} \mathrm{~L}^{-1}\right)$. All medium components were separately heat-sterilized $\left(12{ }^{\circ} \mathrm{C}\right)$. The trace element solution contained the following amounts: $\mathrm{FeCl}_{2} \cdot 4 \mathrm{H}_{2} \mathrm{O}, 2 \mathrm{~g}$ $\mathrm{L}^{-1} ; \mathrm{MnCl}_{2} \cdot 4 \mathrm{H}_{2} \mathrm{O}, 3 \mathrm{~g} \mathrm{~L}^{-1} ; \mathrm{ZnSO}_{4} \cdot 6 \mathrm{H}_{2} \mathrm{O}, 3 \mathrm{~g} \mathrm{~L}^{-1} ; \mathrm{CoCl}_{2} \cdot 6 \mathrm{H}_{2} \mathrm{O}, 0.04$ $\mathrm{g} \mathrm{L}^{-1} ; \mathrm{Na}_{2} \mathrm{MoO}_{4} \cdot 2 \mathrm{H}_{2} \mathrm{O}, 0.04 \mathrm{~g} \mathrm{~L}^{-1} ; \mathrm{NiCl}_{2} \cdot 6 \mathrm{H}_{2} \mathrm{O}, 0.5 \mathrm{~g} \mathrm{~L}^{-1}$; and $\mathrm{CuSO}_{4} \cdot 5 \mathrm{H}_{2} \mathrm{O}, 2 \mathrm{~g} \mathrm{~L}^{-1}$. The vitamin solution was filter-sterilized $(0.22$ $\mu \mathrm{m})$ and contained thiamin, $50 \mathrm{mg} \mathrm{L}^{-1}$ ); biotin, $3 \mathrm{mg} \mathrm{L}^{-1}$ ); and cyanocobalamin, $10 \mathrm{mg} \mathrm{L}^{-1}$ ).

Fed-batch fermentation experiments were performed in $5 \mathrm{~L}$ Biostat $\mathrm{B}$ plus bioreactors (with initial $2.5 \mathrm{~L}$ fermentation medium) equipped with controls for $\mathrm{pH}$, temperature, agitation, and dissolved oxygen concentration (DO). The fermentation medium contains glucose, 100 $\mathrm{g} \mathrm{L}^{-1}$; yeast extract, $20 \mathrm{~g} \mathrm{~L}^{-1}$; tryptone, $10 \mathrm{~g} \mathrm{~L}^{-1}$; $\mathrm{KH}_{2} \mathrm{PO}_{4}, 5 \mathrm{~g} \mathrm{~L}^{-1}$; citric acid trisodium salt, $2 \mathrm{~g} \mathrm{~L}^{-1}$; and artificial seawater, $15 \mathrm{~g} \mathrm{~L}^{-1}$. The agitation speed automatically varied from 300 to $800 \mathrm{rpm}$ at a fixed airflow rate of $1.2 \mathrm{vvm}$ to maintain the DO over $20 \%$ air saturation, and the temperature was maintained at $25{ }^{\circ} \mathrm{C}$. A $50 \%(\mathrm{w} / \mathrm{v})$ glucose solution was fed into medium to maintain the glucose concentration at about $15 \mathrm{~g} \mathrm{~L}^{-1}$, and a $50 \%(\mathrm{w} / \mathrm{v})$ yeast extract solution was fed from 10 to $40 \mathrm{~h}$ with a flow rate of $5 \mathrm{~mL} \mathrm{~h}^{-1}$. A computer-controlled watercooling system was added at the air discharge outlet to reduce the evaporation.

Compared with maintaining the $\mathrm{pH}$ by adding $4 \mathrm{~mol} \mathrm{~L}^{-1} \mathrm{HCl}$ in the control group, one kind of SCFA from acetic acid, propanoic acid, isobutyric acid, butanoic acid, isovaleric acid, or pentanoic acid was automatically fed into the medium and controlled the $\mathrm{pH}$ at 6.5 in the treatment groups, because the $\mathrm{pH}$ of the medium gradually rose under the fermentation conditions.

Because some SCFAs such as butanoic acid and pentanoic acid have a strong pungent smell, all of the experiments should be carried out in a ventilated room and the exhaust should be absorbed by a $5 \mathrm{M} \mathrm{NaOH}$ solution and followed by a pillar filled with activated carbon before emission into the environment.

Biomass Determination. The biomass of Aurantiochytrium sp. SD116 was expressed in terms of dry cell weight (DCW). Samples (30 $\mathrm{mL}$ ) of cell suspensions were centrifuged at $7000 \mathrm{~g}$ and $4{ }^{\circ} \mathrm{C}$ for $10 \mathrm{~min}$ after washing twice with $0.2 \mathrm{M}$ phosphate buffer solution. Cell pellets were then freeze-dried to constant weight at $-50{ }^{\circ} \mathrm{C}$ for approximately $60 \mathrm{~h}$.

Lipid Extraction and Fatty Acid Composition Analysis. The total lipid content was calculated using a modified miniaturized BlighDyer method. ${ }^{23}$ Harvested cells were extracted into $100 \mathrm{~mL}$ of chloroform/methanol $(2: 1, \mathrm{v} / \mathrm{v})$ at room temperature. The lipid extract was dried over anhydrous $\mathrm{Na}_{2} \mathrm{SO}_{4}$, and the solvent was removed by evaporation. Afterward, the total lipid was weighed. Fatty acid methyl esters (FAMEs) were prepared according to the method described before. ${ }^{11}$

Preparation of Cell Extracts for Enzymatic Assay. To prepare cell extracts for the enzymatic assay, harvested cells were centrifuged at $7000 \mathrm{~g}$. The pellets were washed with ice-cold distilled water and then rewashed and suspended by a washing buffer [400 mM Tris-HCl buffer, $\mathrm{pH}$ 7.4, 20\% (w/v) glycerol, $1 \mathrm{mM}$ dithioerythritol (DTT)]. After being disrupted by a single pass through a One-Shot cell disrupter (Constant Systems) at $60 \mathrm{MPa}$, the cell lysate was centrifuged $\left(10000 \mathrm{~g}\right.$ for $10 \mathrm{~min}$ at $4{ }^{\circ} \mathrm{C}$ ), and the supernatant was used immediately for the determination of ME activity. Protein concentration was determined using the Bradford method with bovine serum albumin (BSA) as a standard.

$\mathrm{NADP}^{+}-$Malic Enzyme Assay. Activity of malic enzyme was determined spectrophotometrically by monitoring the rate of NADPH formation at $25{ }^{\circ} \mathrm{C}$. The reaction was assayed in a $1.0 \mathrm{~mL}$ reaction volume containing $50 \mathrm{mM}$ Tris- $\mathrm{HCl}(\mathrm{pH} 7.4), 0.5 \mathrm{mM} \mathrm{NADP}{ }^{+}, 20$ $\mathrm{mM}$ L-malate, and $10 \mathrm{mM} \mathrm{MgCl}_{2}{ }^{24} \mathrm{~A}$ unit of enzyme activity (U) was defined as the formation of the quantitative amount of NADPH per minute, which was equivalent to an increase in $\mathrm{OD}_{340}$ of $0.001 / \mathrm{min}$. Specific activity (U/mg protein) was defined as the units of activity per milligram of protein. ${ }^{25}$

Glucose Assay. Five milliliter samples were taken from the fermentor every $4 \mathrm{~h}$, and the residual glucose was analyzed by a biosensor equipped with glucose oxidase electrode (SBA-40E, Institute of Biology, Shandong Academy of Sciences, China). ${ }^{26}$

\section{RESULTS AND DISCUSSION}

Impact of Short-Chain Fatty Acids on Cell Growth and Lipid Accumulation of Aurantiochytrium sp. SD116. A total volume of $314 \mathrm{~mL}$ of acetic acid, $327 \mathrm{~mL}$ of propanoic acid, $275 \mathrm{~mL}$ of isobutyric acid, $282 \mathrm{~mL}$ of butanoic acid, 241 $\mathrm{mL}$ of isovaleric acid, or $249 \mathrm{~mL}$ of pentanoic acid was fed into the medium during the fermentation individually, and no or 

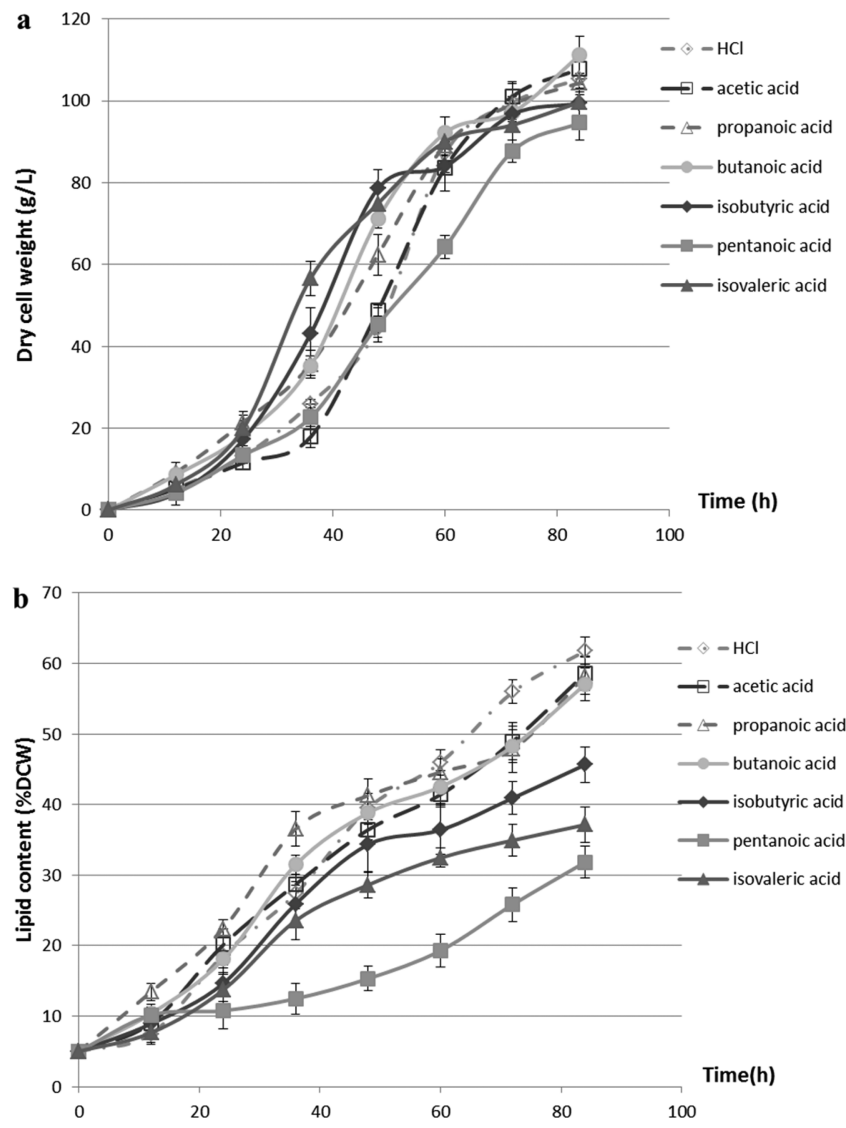

Figure 1. Impact of short-chain fatty acids on the cell growth (a) and lipid accumulation (b) in fermentation of Aurantiochytrium sp. SD116.

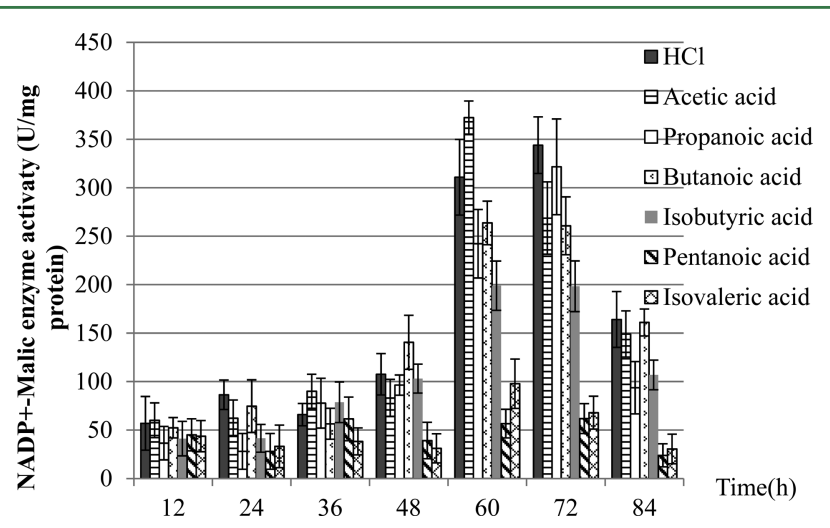

Figure 2. Impact of short-chain fatty acids on the malic enzyme activities in fermentation of Aurantiochytrium sp. SD116.

traces of SCFA were detected from the zymotic fluid by nuclear magnetic resonance (NMR) at the end of the fermentation (data not shown). This indicated that all SCFAs tested in this study could be fully utilized by Aurantiochytrium sp. SD116 during fermentation. The biomass, total glucose consumption, and total acid consumption after the whole fermentation are shown in Table 1. When the SCFA was fed, the total glucose consumption was reduced, but the biomass was only slightly changed. This phenomenon suggested this strain could use all of these SCFAs preferentially as a supplementary carbon resource. However, the percent conversion from each mole carbon to biomass was reduced from 10.4 to about $8 \mathrm{~g} \mathrm{DCW} /$ mol carbon.
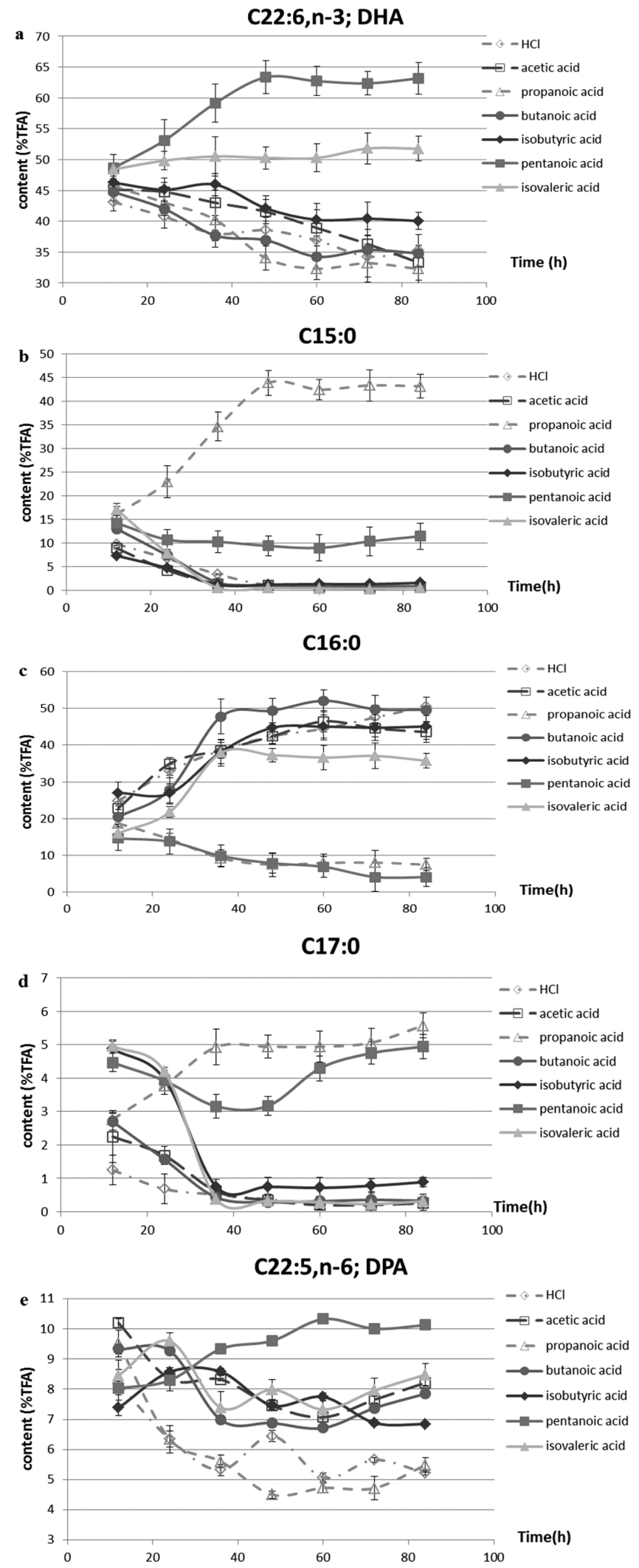

Figure 3. Changes of major fatty acids in Aurantiochytrium sp. SD116 during the fermentation ( $a$, DHA variation; $b$, pentadecanoic acid variation; c, hexadecanoic acid variation; $d$, heptadecanoic acid variation; e, DPA variation).

A treatment with $\mathrm{HCl}$ for $\mathrm{pH}$ adjustment instead of SCFA was set up as the control group. As shown in Figure 1a, all 
Table 2. Fatty Acid Composition of Aurantiochytrium sp. SD116 in Each Group after the 84 h Fermentation

\begin{tabular}{|c|c|c|c|c|c|c|c|}
\hline \multirow[b]{2}{*}{ fatty acid } & \multicolumn{7}{|c|}{ fatty acid contents ${ }^{a}(\%)$} \\
\hline & $\mathrm{CK}$ & A & $\mathrm{P}$ & B & IB & V & IV \\
\hline C14:0 & 3.44 & 6.447 & 2.98 & 3.675 & 2.602 & 1.431 & 1.094 \\
\hline C14:1 & 0.371 & 0.971 & 0.571 & 0.209 & 0.151 & 0.462 & 0.171 \\
\hline C15:0 & 0.693 & 0.693 & 43.224 & 0.897 & 1.613 & 11.803 & 0.516 \\
\hline C16:0 & 50.559 & 43.559 & 7.437 & 49.627 & 45.09 & 5.139 & 35.741 \\
\hline C16:1 & 0.211 & 0.711 & 0.502 & 0.109 & 0.161 & 0.616 & 0.142 \\
\hline C17:0 & 0.28 & 0.258 & 5.576 & 0.33 & 0.888 & 4.755 & 0.316 \\
\hline C18:0 & 1.219 & 2.569 & 0.413 & 1.471 & 1.893 & 0.626 & 1.285 \\
\hline C18:1 & 0.215 & 0.488 & 0.34 & 0.181 & 0.149 & 0.496 & 0.115 \\
\hline C18:3 & 0.311 & 0.51 & 0.464 & 0.484 & 0.328 & 0.528 & 0.122 \\
\hline $\mathrm{C} 20: 5(\mathrm{n}-3)$ & 0.33 & 0.53 & 0.368 & 0.22 & 0.305 & 0.469 & 0.123 \\
\hline$C 22: 5(n-6)$ & 7.017 & 8.217 & 5.486 & 7.852 & 6.839 & 12.316 & 8.487 \\
\hline$C 22: 6(n-3)$ & 35.298 & 33.298 & 32.267 & 34.808 & 40.064 & 61.4 & 51.772 \\
\hline
\end{tabular}

SCFA-treated groups achieved similar biomass in comparison with the control group (ca. $100 \mathrm{~g} \mathrm{~L}^{-1} \mathrm{DCW}$ ). The growth of all groups reached exponential growth phase in $40 \mathrm{~h}$ and reached stable phase at about $80 \mathrm{~h}$. This suggested that SCFA had little effect on the growth of Aurantiochytrium. However, the effect of SCFA on lipid accumulation varied (Figure $1 \mathrm{~b}$ ). After $84 \mathrm{~h}$ of fermentation, the lipid content of the control group was $61.7 \%$ of DCW, and the acetic acid, propanoic acid, and butanoic acid treatment groups showed slightly less lipid accumulation. Nevertheless, the lipid contents of the pentanoic acid treatment group and the isovaleric acid treatment group were significantly decreased to 31.8 and $37.1 \%$ of DCW, respectively. These results demonstrated that along with the carbon chain elongation of SCFA, especially pentanoic acid and isovaleric acid, the lipid accumulation was obviously restrained and reduced sharply.

To explain the phenomenon, the three key factors affecting the lipid accumulation should be primarily considered, that is, the supply of acyl-CoA, ATP, and NADPH. ${ }^{18}$ Because the cell growth was little influenced by the treatments of SCFAs, it was difficult to envisage a deficiency of ATP and acetyl-CoA or acylCoA supply for the lipid biosynthesis. Thus, the major factor that affected the fatty acid biosynthesis in the treatment groups should be the supply of reducing power NADPH. It has been suggested that the function of malic enzyme in lipid biosynthesis is to supply NADPH for fatty acid synthase, whereas other NADPH-generating enzymes, such as $\mathrm{NADP}^{+}$dependent isocitrate dehydrogenase, glucose-6-phosphate dehydrogenase, and 6-phosphogluconate dehydrogenase, play only a slight role in the NADPH supply for lipid accumulation. $^{21,27,28}$ To characterize the generation of $\mathrm{NADPH}$ in the treatment groups in this study, the activity of malic enzymes in all treatment groups was investigated. As shown in Figure 2, except for pentanoic acid and isovaleric acid treatment groups, the malic enzyme activities in the other groups all had similar variation tendencies. At the early lipid accumulation stage (fermentation before $48 \mathrm{~h}$ ), little lipid was accumulated in this stage, and all of the ME activities were all lower than $110 \mathrm{U} / \mathrm{mg}$ protein. Subsequently, the ME activities were notably increased to more than $250 \mathrm{U} / \mathrm{mg}$ protein conjugated large accumulation of lipid at the rapid lipid biosynthesis stage (from 48 to $80 \mathrm{~h}$ ). During the whole fermentation period, the ME activities in the pentanoic acid treatment group were all lower than $70 \mathrm{U} / \mathrm{mg}$ protein, indicating an insufficient $\mathrm{NADPH}$ supply, which finally led to less lipid accumulation in cells. These data indicated that the reduced lipid accumulation in pentanoic acid and isovaleric acid treated groups was caused by the insufficient supply of $\mathrm{NADPH}$, and the malic enzyme activity was important for the accumulation of storage lipid in Aurantiochytrium.

Impact of Short-Chain Fatty Acids on the Fatty Acids Composition of Aurantiochytrium sp. SD116. The fatty acid composition of Aurantiochytrium sp. SD116 was detected in all treatment groups, and significant changes were observed due to the supplementation of various SCFAs. The changes of major fatty acids in Aurantiochytrium sp. SD116 during the fermentation are shown in Figure 3, and the fatty acid composition of this strain in each group after the $84 \mathrm{~h}$ of fermentation is shown in Table 2. When even-carbon-chain fatty acids (acetic acid, butanoic acid, and isobutyric acid) were fed into the fermentation system, the fatty acid compositions were similar to that of the control group, because the additives were metabolized to acetyl-CoA or probably butyryl-CoA. However, when odd-carbon-chain fatty acids (propanoic acid, pentanoic acid, and isovaleric acid) were fed into the medium, the fatty acid compositions were drastically changed.

With the supplementation of propanoic acid, the percentages of odd long-chain fatty acids (pentadecanoic acid, C15:0; and heptadecanoic acid, C17:0) in cells were sharply increased, especially that of pentadecanoic acid, with the highest level of over $43 \%$ of total fatty acids. This phenomenon was probably caused by converting propanoic acid to propionyl-CoA, which was subsequently utilized by the fatty acid synthase in cells. The DHA contents of total fatty acids in the pentanoic acid and isovaleric acid treatment groups, 63.4 and $51.8 \%$, respectively, were extremely higher than those in the other treatment groups and the control group, which were between 32.3 and $40.4 \%$. This result illustrated that the pentanoic acid or isovaleric acid treatment reduced the anabolism of the FAS pathway, probably by inhibiting the ME activity (Figure 2), whetrsd the PKS pathway was not affected. This demonstrated that the NADPH needed for the PKS pathway should come from other NADPHgenerating enzymes, because the yields of the polyunsaturated fatty acids, DPA and DHA, biosynthesized from the PKS pathway were not decreased along with the low ME activity.

A small yield ( $<1 \%$ of TFA) of a branched-chain fatty acid, methyl 13-methyltetradecanoate (Supporting Information Figure S1), was detected in the isovaleric acid treatment group, whereas no branched-chain fatty acid was discovered in other treatment groups, including the isobutyric acid treatment 
$\mathbf{a}$
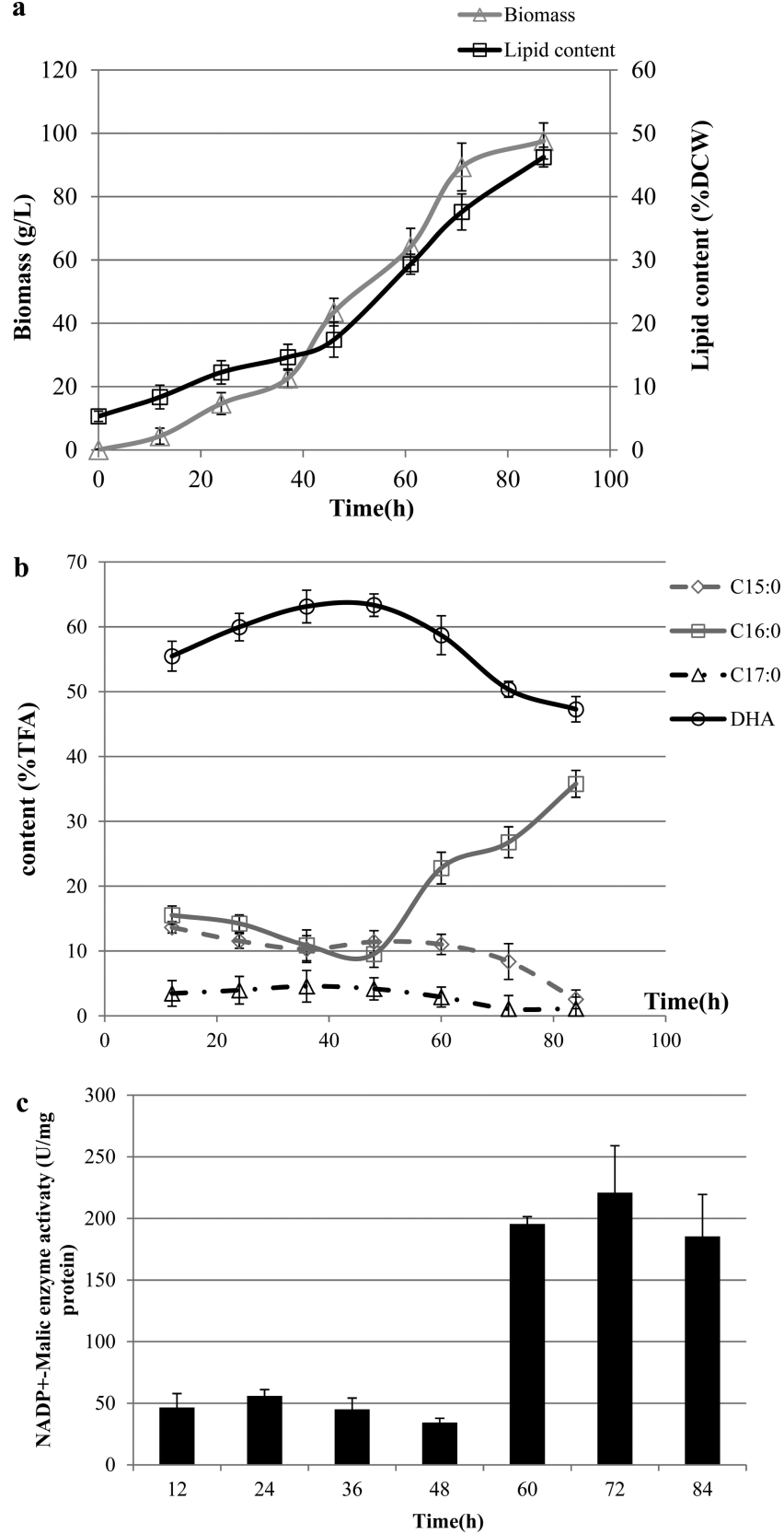

Figure 4. Reversible impact of pentanoic acid on the lipid accumulation (a), fatty acid composition (b), and malic enzyme activity (c) in fermentation of Aurantiochytrium sp. SD116.

group. This indicated that the branched SCFAs were hardly utilized directly by the fatty acid synthase. Instead, they were largely metabolized and probably converted into acetyl-CoA for fatty acid synthesis.

The results of this section demonstrate that the fatty acid synthase of Aurantiochytrium, which mainly charged for the synthesis of saturated fatty acids, could utilize many kinds of acyl-CoA, whereas the polyketide synthase could use only acetyl-CoA as the substrate, because no other long-chain polyunsaturated fatty acid was found except DPA and DHA. In combination with the results of section 3.1, one could conclude that the FAS and PKS pathways utilize different acyl-CoA as substrates and possess independent sources of NADPH, which may provide a new strategy to enhance DHA production. At the middle and later periods of fermentation, inhibiting the ME activity or promoting the other NADPH-generating enzyme activities, which could be achieved by certain additive or genetic engineering, may greatly increase the final DHA proportion in total fatty acids.

Reversible Impact on the Lipid Accumulation and Fatty Acid Composition of Aurantiochytrium by the Pentanoic Acid Treatment. A blocking experiment was carried out to test the impact reversibility of the pentanoic acid treatment. The pentanoic acid stress was removed at $40 \mathrm{~h}$, and then the $\mathrm{pH}$ was controlled by $\mathrm{HCl}$. As shown in Figure 4a,b, the ME activities were rapidly increased to $220.94 \mathrm{U} / \mathrm{mg}$ protein after the stress was removed, and the rate of lipid accumulation was promoted from 0.363 to $0.801 \mathrm{~g} \mathrm{~L}^{-1} \mathrm{~h}^{-1}$. The fatty acid compositions of Aurantiochytrium sp. SD116 were also drastically changed (Figure 4c). The DHA content was changed to $47.29 \%$ of TFA at the end of fermentation from $63.35 \%$ of TFA at $48 \mathrm{~h}$, and the hexadecanoic acid (C16:0) was increased from 9.53 to $35.79 \%$ of TFA. These changes indicated that the increasing ME activity after the removal of pentanoic acid stress quickly strengthened the fatty acid synthase pathway, which caused the hexadecanoic acid accumulation in cells. In summary, short-chain fatty acids can reversibly affect the lipid accumulation and the fatty acid composition of Aurantiochytrium sp. SD116.

The results from this study provided new insight into the fatty acid synthesis in Aurantiochytrium. First, the FAS and PKS in Aurantiochytrium can utilize different acyl-CoA as substrates and possess independent sources of NADPH. Second, the activity of malic enzyme only couples to the FAS pathway for the saturated fatty acid biosynthesis by supplying the reducing power NADPH, whereas the reducing power for the PKS pathway to synthesize the PUFA should come from other NADPH-generating enzymes. These new findings provide new clues to the improvement of DHA production by Aurantiochytrium. The different substrate utilizations and NADPH sources by the two pathways could be used to suppress SFA synthesis or increase DHA production of Aurantiochytrium through either development of specific inhibitors or genetic engineering in the future.

\section{ASSOCIATED CONTENT}

\section{Supporting Information}

Identification of the branched-chain fatty acid methyl 13methyltetradecanoate. This material is available free of charge via the Internet at http://pubs.acs.org.

\section{AUTHOR INFORMATION}

\section{Corresponding Authors}

*(X.S.) Mailing address: No. 189 Songling Road, Laoshan District, Qingdao 266101, China. Phone:+86 53280662705. Fax: +86532 80662707. E-mail: songxj@qibebt.ac.cn.

*(Q.C.) Mailing address: No. 189 Songling Road, Laoshan District, Qingdao 266101, China. Phone: +86 53280662706. Fax: +86532 80662707. E-mail: cuiqiu@qibebt.ac.cn.

\section{Author Contributions}

${ }$ X.S. and Y.T. contributed equally to this work.

\section{Funding}

This study was supported by the Knowledge Innovation Program of the Chinese Academy of Sciences (KSCX2-EWJ10) and the National High Technology Research and Development Program of China (2011BAD22B02). 


\section{Notes}

The authors declare no competing financial interest.

\section{REFERENCES}

(1) Innis, S. M. Dietary omega 3 fatty acids and the developing brain. Brain Res. 2008, 1237, 35-43.

(2) Hajjaji, N.; Schubnel, V.; Bougnoux, P. Determinants of DHA incorporation into tumor tissue during dietary DHA supplementation. Lipids 2011, 46, 1063-1069.

(3) Giltay, E. J.; Geleijnse, J. M.; Kromhout, D. Effects of n-3 fatty acids on depressive symptoms and dispositional optimism after myocardial infarction. Am. J. Clin. Nutr. 2011, 94, 1442-1450.

(4) Graham, I. A.; Larson, T.; Napier, J. A. Rational metabolic engineering of transgenic plants for biosynthesis of omega-3 polyunsaturates. Curr. Opin. Biotechnol. 2007, 18, 142-147.

(5) Ratledge, C. Fatty acid biosynthesis in microorganisms being used for single cell oil production. Biochimie 2004, 86, 807-815.

(6) Mariana Rosa, S.; Abel Soria, M.; Guillermo Velez, C.; Angel Galvagno, M. Improvement of a two-stage fermentation process for docosahexaenoic acid production by Aurantiochytrium limacinum SR21 applying statistical experimental designs and data analysis. Bioresour. Technol. 2010, 101, 2367-2374.

(7) Gao, M.; Song, X. J.; Feng, Y. G.; Li, W. L.; Cui, Q. Isolation and characterization of Aurantiochytrium species: high docosahexaenoic acid (DHA) production by the newly isolated microalga, Aurantiochytrium sp SD116. J. Oleo Sci. 2013, 62, 143-151.

(8) Kim, K.; Kim, E. J.; Ryu, R.-G.; Park, S.; Choi, Y.-E.; Yang, J.-W. A novel fed-batch process based on the biology of Aurantiochytrium sp. KRS101 for the production of biodiesel and docosahexaenoic acid. Bioresour. Technol. 2013, 135, 269-274.

(9) Yaguchi, T.; Tanaka, S.; Yokochi, T.; Nakahara, T.; Higashihara, T. Production of high yields of docosahexaenoic acid by Schizochytrium sp. strain SR21. J. Am. Oil Chem. Soc. 1997, 74, 1431-1434.

(10) Ganuza, E.; Anderson, A. J.; Ratledge, C. High-cell-density cultivation of Schizochytrium sp. in an ammonium/pH-auxostat fedbatch system. Biotechnol. Lett. 2008, 30, 1559-1564.

(11) Song, X. J.; Zhang, X. C.; Kuang, C. H.; Zhu, L. Y.; Guo, N. Optimization of fermentation parameters for the biomass and DHA production of Schizochytrium limacinum OUC88 using response surface methodology. Process Biochem. 2007, 42, 1391-1397.

(12) Ren, L. J.; Feng, Y.; Li, J.; Qu, L.; Huang, H. Impact of phosphate concentration on docosahexaenoic acid production and related enzyme activities in fermentation of Schizochytrium sp. Bioprocess Biosyst. Eng. 2012, DOI: 10.1007/s00449-012-0844-8.

(13) Huang, T. Y.; Lu, W. C.; Chu, I. M. A fermentation strategy for producing docosahexaenoic acid in Aurantiochytrium limacinum SR21 and increasing C22:6 proportions in total fatty acid. Bioresour. Technol. 2012, 123, 8-14.

(14) Metz, J. G.; Roessler, P.; Facciotti, D.; Levering, C.; Dittrich, F.; Lassner, M.; Valentine, R.; Lardizabal, K.; Domergue, F.; Yamada, A.; Yazawa, K.; Knauf, V.; Browse, J. Production of polyunsaturated fatty acids by polyketide synthases in both prokaryotes and eukaryotes. Science 2001, 293, 290-293.

(15) Durnford, E.; Shahidi, F. Comparison of FA compositions of selected tissues of phocid seals of eastern Canada using one-way and multivariate techniques. J. Am. Oil Chem. Soc. 2002, 79, 1095-1102.

(16) Lippmeier, J. C.; Crawford, K. S.; Owen, C. B.; Rivas, A. A.; Metz, J. G.; Apt, K. E. Characterization of both polyunsaturated fatty acid biosynthetic pathways in Schizochytrium sp. Lipids 2009, 44, 621630.

(17) Hauvermale, A.; Kuner, J.; Rosenzweig, B.; Guerra, D.; Diltz, S.; Metz, J. G. Fatty acid production in Schizochytrium sp involvement of a polyunsaturated fatty acid synthase and a type I fatty acid synthase. Lipids 2006, 41, 739-747.

(18) Hamid, A. A.; Mokhtar, N. F.; Taha, E. M.; Omar, O.; Yusoff, W. M. W. The role of ATP citrate lyase, malic enzyme and fatty acid synthase in the regulation of lipid accumulation in Cunninghamella sp 2A1. Ann. Microbiol. 2011, 61, 463-468.
(19) Katz, L.; Donadio, S. Polyketide synthesis: prospects for hybrid antibiotics. Annu. Rev. Microbiol. 1993, 47, 875-912.

(20) Wynn, J. P.; Hamid, A. B. A.; Ratledge, C. The role of malic enzyme in the regulation of lipid accumulation in filamentous fungi. Microbiology 1999, 145, 1911-1917.

(21) Chang, G.; Luo, Z.; Gu, S.; Wu, Q.; Chang, M.; Wang, X. Fatty acid shifts and metabolic activity changes of Schizochytrium sp. S31 cultured on glycerol. Bioresour. Technol. 2013, 142, 255-260.

(22) Sijtsma, L. Alternative carbon sources for heterotrophic production of DHA by the marine alga Crypthecodinium cohnii. In Single Cell Oils, 2nd ed.; Ratledge, C., Ed.; AOCS Press: Champaign, IL, USA, 2010; pp 131-149.

(23) Bligh, E. G.; Dyer, W. J. A rapid method of total lipid extraction and purification. Can. J. Biochem. Physiol. 1959, 37, 911-917.

(24) Rodriguez, E.; Navone, L.; Casati, P.; Gramajo, H. Impact of malic enzymes on antibiotic and triacylglycerol production in Streptomyces coelicolor. Appl. Environ. Microbiol. 2012, 78, 4571-4579.

(25) Ren, L. J.; Huang, H.; Xiao, A. H.; Lian, M.; Jin, L. J.; Ji, X. J. Enhanced docosahexaenoic acid production by reinforcing acetyl-CoA and NADPH supply in Schizochytrium sp. HX-308. Bioprocess Biosyst. Eng. 2009, 32, 837-843.

(26) Lian, M.; Huang, H.; Ren, L.; Ji, X.; Zhu, J.; Jin, L. Increase of docosahexaenoic acid production by Schizochytrium sp. through mutagenesis and enzyme assay. Appl. Biochem. Biotechnol. 2010, 162, 935-941.

(27) Zhang, Y.; Adams, I. P.; Ratledge, C. Malic enzyme: the controlling activity for lipid production? Overexpression of malic enzyme in Mucor circinelloides leads to a 2.5 -fold increase in lipid accumulation. Microbiology 2007, 153, 2013-2025.

(28) Ratledge, C.; Wynn, J. P. The biochemistry and molecular biology of lipid accumulation in oleaginous microorganisms. Adv. Appl. Microbiol. 2002, 51, 1-51. 